\title{
Tumor Stage-Related Role of Radiotherapy in Patients with an External Auditory Canal and Middle Ear Carcinoma
}

\author{
Jinhyun Choi, MD ${ }^{1}$ \\ Se-Heon Kim, MD, PhD² \\ Yoon Woo Koh, MD, PhD² \\ Eun Chang Choi, MD, PhD² \\ Chang Geol Lee, MD, PhD ${ }^{3}$ \\ Ki Chang Keum, MD, PhD ${ }^{3}$
}

${ }^{1}$ Department of Radiation Oncology,

Gangnam Severance Hospital,

Yonsei University College of Medicine, Seoul,

${ }^{2}$ Department of Otorhinolaryngology,

Yonsei University College of Medicine, Seoul,

${ }^{3}$ Department of Radiation Oncology,

Yonsei Cancer Center, Yonsei University

College of Medicine, Seoul, Korea

\section{Purpose}

The purpose of this study was to evaluate the clinical outcomes of patients treated with radiotherapy (RT) for a carcinoma of the external auditory canal (EAC) and middle ear.

\section{Materials and Methods}

The records of 32 patients who received RT from 1990 to 2013 were reviewed retrospectively. The Pittsburgh classification was used to stage all the cancers (early stage, T1/T2 [n=12]; advanced stage, T3/T4 or N positive [n=20]). Twenty-one patients (65.6\%) were treated with postoperative RT and 11 patients (34.4\%) were treated with definitive RT. The median radiation doses for postoperative and definitive RT were 60 Gy and 64.8 Gy, respectively. Chemotherapy was administered to seven patients (21.9\%).

\section{Results}

The 5-year overall survival and disease-free survival rates for all patients were $57 \%$ and $52 \%$, respectively. The disease control rates for the patients with early stage versus advanced stage carcinoma were 55.6\% (5/9) and 50\% (6/12) in the postoperative RT group and $66.7 \%(2 / 3)$ and $37.5 \%$ (3/8) in the definitive RT group, respectively. Overall, 15 cases (14 patients, 46.7\%) experienced treatment failure; these failures were classified as local in four cases, regional in one case, and distant in 10 cases. The median follow-up period after RT was 51 months (range, 7 to 286 months).

\section{Conclusion}

Patients with early stage carcinoma achieved better outcomes when definitive RT was used. Advanced stage carcinoma patients experienced better outcomes with postoperative RT. The high rate of distant failure after RT, with or without surgery, reflected the lack of a consensus regarding the best therapeutic approach for treating carcinoma of the EAC and middle ear.
Department of Radiation Oncology,

Yonsei Cancer Center, Yonsei University

College of Medicine, 50-1 Yonsei-ro,

Seodaemun-gu, Seoul 03722, Korea

Tel: 82-2-2228-8112

Fax: 82-2-312-9033

E-mail: KCKEUM@yuhs.ac

Received April 20, 2016

Accepted June 3, 2016

Published Online July 4, 2016
Key words

Radiotherapy, Ear canal, Middle ear

\section{Introduction}

Cancer of the external auditory canal (EAC) and middle ear is rare, with a prevalence of one to six patients per 1 million people [1-3]. The disease presents variable patterns of clinical practice in terms of management. Although several treatment modalities have been investigated, there are no standard guidelines for treating cancers of the EAC and middle ear due to a lack of prospective randomized studies. A surgical resection with a clear tumor margin is usually recommended for resectable disease [4]. On the other hand, the role of adjuvant and definitive radiotherapy (RT) in treating unresectable disease is less well-defined.

The prognosis of an EAC carcinoma depends on the disease stage and the primary treatment approach used [5]. Investigators have reported the 5-year survival rates ranging from $10 \%$ for advanced stage to as high as $85 \%$ for early stage disease [4,6-9]. In the literature, the 5-year locoregional control rates range from $20 \%$ for advanced stage to $70 \%$ for early 
stage disease [10]. As there is no widely accepted staging system, using the results from different reports to compare the treatment outcomes can be difficult $[3,11]$. Moreover, although several articles describe the efficacy of RT for EAC tumors, there is little information on the relationships between the extent of disease and the clinical outcomes for different types of RT $[4,12,13]$. To help fill this void, this study evaluated the treatment outcomes for 32 patients with a carcinoma of the EAC and middle ear who were treated with postoperative or definitive RT, with or without chemotherapy, at the authors' institution over a 20-year period.

\section{Materials and Methods}

\section{Patients}

The records of 32 patients treated with postoperative or definitive RT at the Yonsei Cancer Center for primary carcinoma of the EAC and middle ear between 1990 and 2013 were reviewed retrospectively. The Institutional Review Board of Severance Hospital, Korea (IRB No. 4-2015-0884) approved this retrospective study in accordance with the ethical guidelines and the Declaration of Helsinki. Initially, all patients had previously untreated primary cancer. All patients had received computed tomography (CT) or magnetic resonance imaging (MRI) scans, or both, prior to treatment. The tumor stage was established according to the University of Pittsburgh system [14]. Four, eight, five, and 15 patients were classified as having stage I (T1N0), stage II (T2N0), stage III (T3N0), and stage IV (T3N1, T4N0, T4N1) carcinoma, respectively. Early stage carcinoma and advanced stage carcinoma was defined as T1/T2 ( $\mathrm{n}=12)$ and T3/T4 or $N$ positive $(n=20)$, respectively. Four patients had regional node involvement $(12.5 \%)$. Surgical procedures were chosen at the discretion of the surgeon in accordance with the tumor extent and nodal status. Twenty-one patients $(65.6 \%)$ were treated surgically. Six of these patients received a total or subtotal temporal bone resection (TBR), eight received a partial or lateral TBR, and seven received a wide excision. A neck dissection was performed only in patients with clinically positive neck nodes. Postoperative RT was used to treat $21(65.6 \%)$ patients and definitive RT was used to treat 11 $(34.4 \%)$. Chemotherapy was administered to seven patients $(21.9 \%)$.

All patients were treated using external beam RT with curative intent. With the exception of two patients treated before the year 2000, all patients underwent CT-based simulation. The structures were contoured manually on the CT scan slices. An immobilization device was used during RT. In the postoperative setting, radiation was given once per day at 1.8 to $2.25 \mathrm{~Gy}$. The total doses applied to the primary tumor bed were 59.4 or $60 \mathrm{~Gy}$ for patients with negative tumor margins and between 54 and 71.4 Gy for those patients with positive tumor margins. The prescribed doses in definitive RT were 51 to 75.6 Gy at 1.8 to 2.5 Gy per fraction for the primary tumor bed. Based on the pretreatment CT or MRI scan results, the treatment volumes were drawn to include the primary tumor bed or ipsilateral lymph nodes, or both, with 1-2 cm margins. No patient received prophylactic clinically negative neck irradiation. Most patients were treated using three or more fields. Two patients received treatment using a wedge-pair field arrangement with megavoltage irradiation. Eleven patients $(34.4 \%)$ were treated with intensitymodulated RT.

\section{Statistical analysis}

The differences in patient characteristics between the two radiation groups were assessed using a chi-square test. The overall survival (OS) rates, disease-free survival (DFS) rates, disease control rates, and failure patterns were evaluated. The survival time was defined as the interval from the date of the completion of RT to the date of the last follow-up or death. The survival data were analyzed using the KaplanMeier method. The sites of failure were recorded as local, regional, or distant. Statistical analysis was carried out using SPSS ver. 18.0 (SPSS Inc., Chicago, IL). A $\mathrm{p} \leq 0.05$ was considered significant.

\section{Results}

\section{Patient characteristics}

Table 1 lists the patient and tumor characteristics. The median age was 51 years (range, 26 to 87 years) at the time of diagnosis. Twenty patients were male, and 12 patients were female. The primary tumor site was the EAC and middle ear in 31 and one patient, respectively. A histological examination revealed squamous cell carcinoma (SCC) in 21 patients $(65.6 \%)$. The other histological types present in the other patients were adenoid cystic carcinoma (ACC, 28.1\%), adenocarcinoma (3.1\%), and malignant melanoma (3.1\%). Most of the patients had Eastern Cooperative Oncology Group performance scores of 0 or 1 .

The most frequent symptoms and signs were otorrhea ( $\mathrm{n}=12$, including two patients with hemorrhagic otorrhea), preauricular mass $(n=8)$, otalgia $(n=7)$, and facial palsy $(n=5)$. Two patients complained of hearing loss. An analysis of the 
Table 1. Comparison of the patient and tumor characteristics according to the type of radiotherapy

\begin{tabular}{|c|c|c|c|}
\hline Characteristic & Total & $\begin{array}{l}\text { Postoperative RT } \\
\qquad(\mathrm{n}=21)\end{array}$ & $\begin{array}{c}\text { Definitive RT } \\
\qquad(n=11)\end{array}$ \\
\hline \multicolumn{4}{|l|}{ Sex } \\
\hline Male & $20(62.5)$ & 12 & 8 \\
\hline Female & $12(37.5)$ & 9 & 3 \\
\hline \multicolumn{4}{|l|}{ Age (yr) } \\
\hline$\leq 50$ & $15(46.9)$ & 10 & 5 \\
\hline$>50$ & $17(53.1)$ & 11 & 6 \\
\hline \multicolumn{4}{|l|}{ Location } \\
\hline EAC & $31(96.9)$ & 20 & 11 \\
\hline $\mathrm{ME}$ & $1(3.1)$ & 1 & 0 \\
\hline \multicolumn{4}{|l|}{ Stage } \\
\hline I-II & $12(37.5)$ & 9 & 3 \\
\hline III-IV & $20(62.5)$ & 12 & 8 \\
\hline \multicolumn{4}{|l|}{ Histologic type } \\
\hline SCC & $21(65.6)$ & 11 & 10 \\
\hline ACC & $9(28.1)$ & 8 & 1 \\
\hline Others & $2(6.3)$ & 2 & 0 \\
\hline \multicolumn{4}{|l|}{ Total dose (Gy) } \\
\hline$\leq 60$ & $15(46.9)$ & 12 & 3 \\
\hline$>60$ & $17(53.1)$ & 9 & 8 \\
\hline \multicolumn{4}{|l|}{ Resection margin } \\
\hline Positive & $15(71.4)$ & 15 & - \\
\hline Negative & $6(28.6)$ & 6 & - \\
\hline RT-related toxicity & $5(15.6)$ & 4 & 1 \\
\hline Follow-up (mo) & $51(7-286)$ & & \\
\hline
\end{tabular}

Values are presented as number $(\%)$ or median (range). RT, radiotherapy; EAC, external auditory canal; ME, middle ear; SCC, squamous cell carcinoma; ACC, adenoid cystic carcinoma.

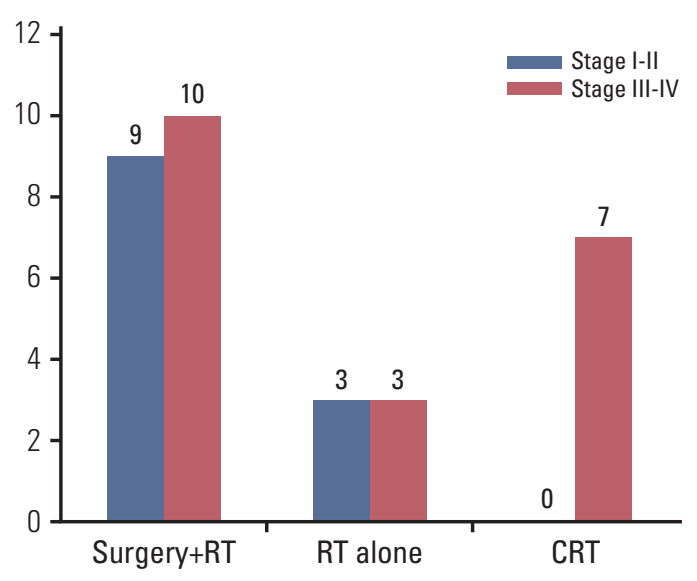

Fig. 1. Patterns of care for 32 patients with external auditory canal and middle ear carcinoma according to the tumor stage. RT, radiotherapy; CRT, chemoradiation therapy. treatment patterns showed that 59\% $(\mathrm{n}=19)$ of the patients had been treated with combined surgery and RT, 22\% ( $\mathrm{n}=7)$ with chemoradiotherapy, and 19\% ( $n=6)$ with RT alone. Nine of the 12 patients with stage I-II disease received surgery with RT; three patients received RT alone. None the patients with stage I-II carcinoma received chemotherapy. Ten of the 20 patients with stage III-IV disease underwent surgery with RT; seven underwent combined chemoradiotherapy and three were treated using RT alone (Fig. 1). Concurrent chemoradiotherapy was delivered only to those patients with stage III-IV disease.

\section{Treatment outcomes}

The median post-RT follow-up period was 51 months (range, 7 to 286 months). Twenty-four of the 32 patients (75\%) experienced a complete response after RT; five patients $(15.6 \%)$ had a partial response. The 5-year OS and DFS rates for all patients were $57 \%$ and $52 \%$, respectively. Fig. 2 presents the survival outcomes according to the stage and RT 

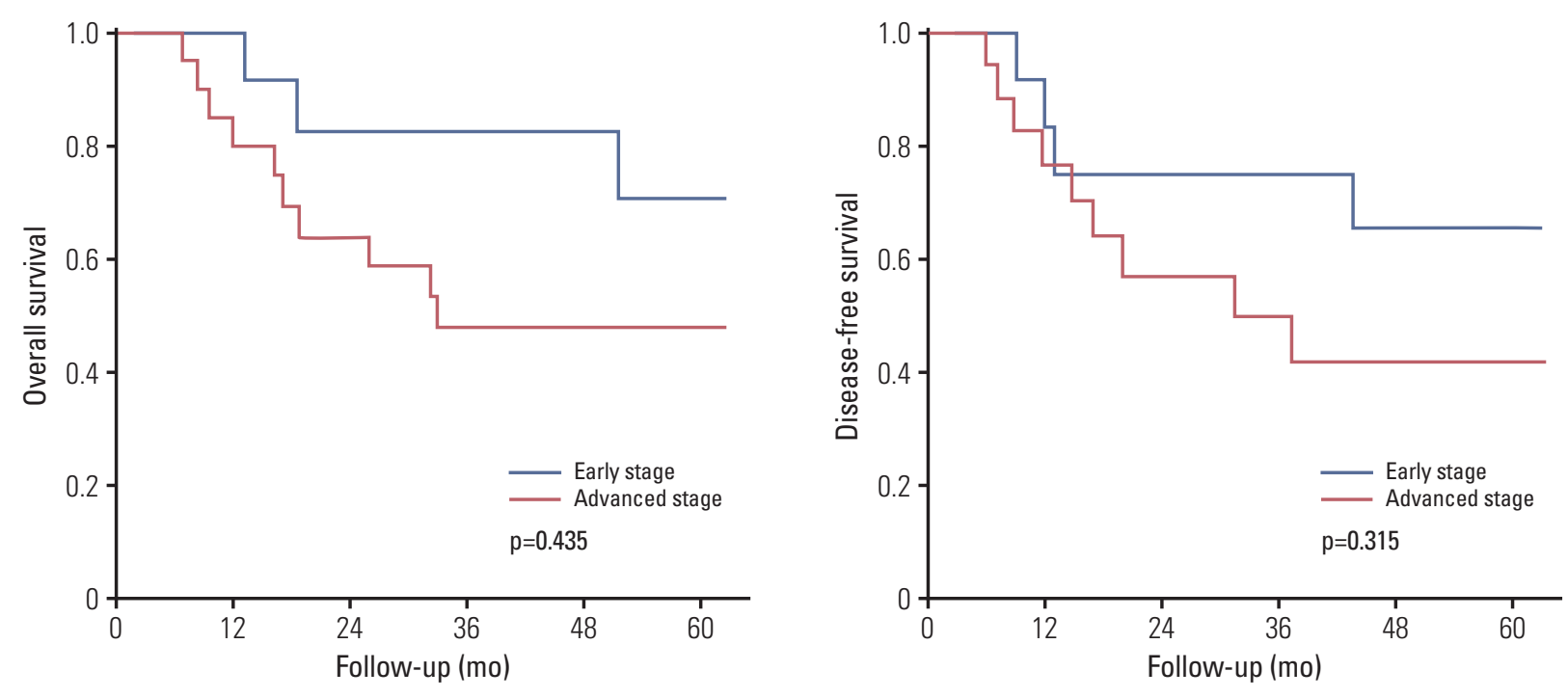

B
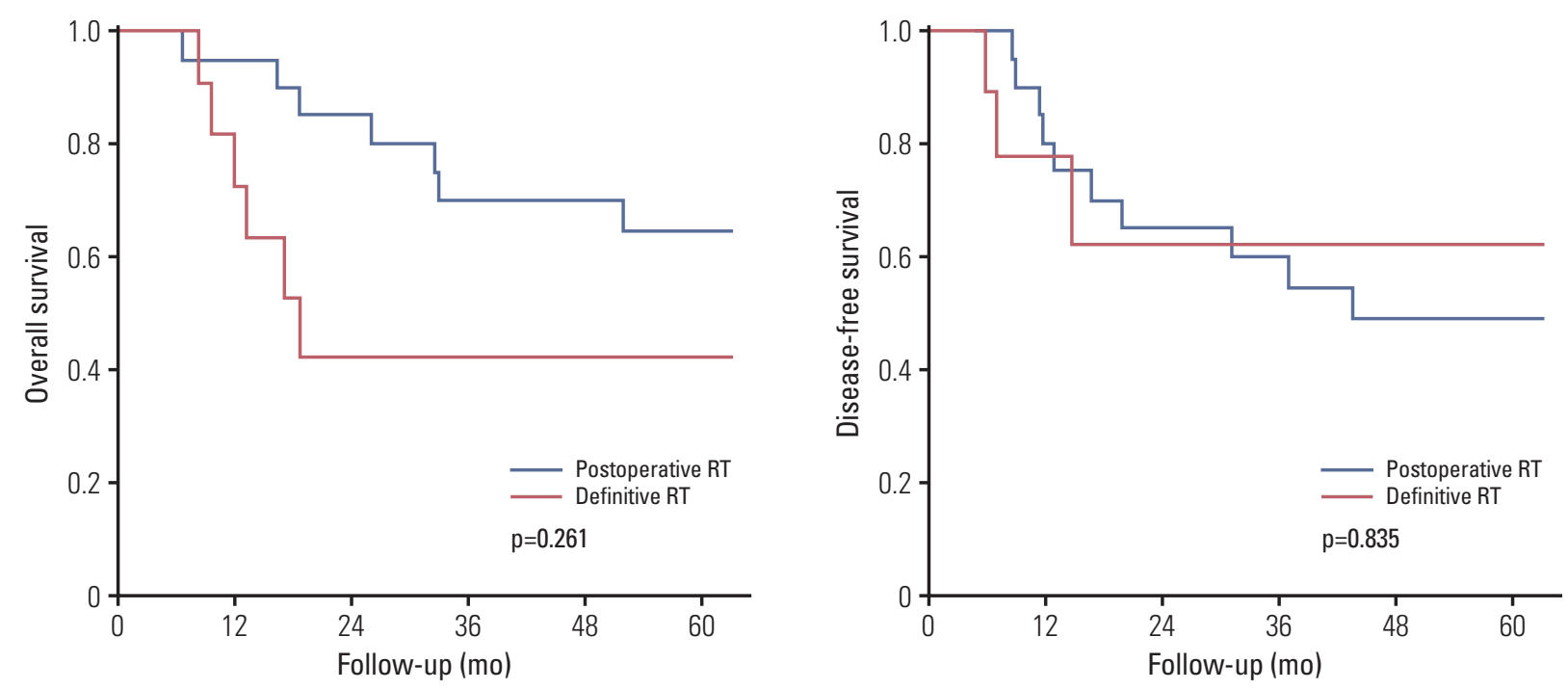

Fig. 2. Overall survival and disease-free survival rates for patients with early stage versus advanced stage external auditory canal and middle ear carcinoma (A) and for treatment with postoperative radiotherapy (RT) vs. definitive RT (B).

type. The 5-year OS and DFS rates for early stage versus advanced stage disease were $70.7 \%$ versus $48 \%(\mathrm{p}=0.315)$ and $65.6 \%$ versus $41.4 \%$ ( $\mathrm{p}=0.435)$, respectively. The 5 -year OS rate was $64.8 \%$ and $42.4 \%$ for the postoperative and definitive RT group, respectively ( $\mathrm{p}=0.261$ ). The 5 -year DFS rate was $49.1 \%$ for the postoperative RT group and $62.2 \%$ for the definitive RT group ( $\mathrm{p}=0.835)$. No patient experienced grade 3 or higher acute or chronic radiation-induced toxicity.

Table 2 lists the stage-related disease control rates. Sixteen patients $(50 \%)$ showed no evidence of a disease status with- out recurrence at the last follow-up visit. The disease control rates for patients with early stage versus advanced stage carcinoma were $55.6 \%(5 / 9)$ versus $50 \%(6 / 12)$ in the postoperative RT group and $66.7 \%(2 / 3)$ versus $37.5 \%(3 / 8)$ in the definitive RT group, respectively. Eleven patients (52.4\%) and five patients $(45.5 \%)$ in the postoperative RT and definitive RT group, respectively, were considered cured. 
Table 2. Stage-related disease control rates for external auditory canal and middle ear cancer patients treated with RT

\begin{tabular}{lrc} 
& Early stage & Advanced stage \\
Postoperative RT & $5 / 9(55.6)$ & $6 / 12(50)$ \\
Definitive RT & $2 / 3(66.7)$ & $3 / 8(37.5)$ \\
Total & $7 / 12(58.3)$ & $9 / 20(45)$ \\
\hline
\end{tabular}

Values are presented as number (\%). RT, radiotherapy.

\section{Patterns of failure}

Two patients experienced disease progression after the initial treatment, and were excluded from the analysis of the failure patterns. Overall, 14 patients $(46.7 \%)$ experienced treatment failure; four of these were classified as local failure and one as regional failure. Ten distant failures were observed as the first site of recurrence. The most common metastatic site was the lung (seven cases), followed by the spine (two cases) and bone (one case). One patient had simultaneous lung and spine metastases at the time of failure. Table 3 lists the results of an analysis of the failure patterns according to the RT type. The "any recurrence" rate was $52.4 \%(11 / 21)$ in the postoperative RT group and $44.4 \%$ $(4 / 9)$ in the definitive RT group.

\section{Discussion}

Primary cancer of the EAC and middle ear is extremely rare and tends to be locally advanced at presentation and has a poor prognosis [1]. Surgery (e.g., lateral TBR for T1/T2 disease and subtotal or total TBR for T3/T4 disease) is generally the recommended treatment. In most clinical settings, however, less invasive surgeries are undertaken to treat this disease, even when T3/T4 disease is present, because the EAC is located adjacent to the critical organs, such as the brain and major vessels. Nevertheless, the use of less invasive surgical techniques is the main reason for local failure after surgical treatment [15].

A comparison of the treatment strategies for EAC and middle ear cancer is hindered by treatment and staging classification heterogeneity due to the small patient populations in published series. According to the Pittsburgh staging system [14], standard therapies include lateral TBR or definitive RT for T1 EAC tumors, lateral TBR combined with postoperative RT for T2 tumors, and subtotal or total TBR with postoperative RT for T3 and T4 tumors. On the other hand, despite these standardized treatment recommendations, the prognoses remain poor [15]. At a single institutional experience, seven patients were treated using definitive RT and 22 patients were treated with postoperative RT. Better local and local-regional control was achieved for the early stage (T1/2) tumors than for the late-stage (T3) tumors, even though less than half of the patients were cured [16]. This suggests that a suboptimal treatment approach is being used for the local management of EAC cancers.

The use of meta-analysis is important for an optimal evaluation of the treatment strategies because it has been difficult for a single institution to obtain a sufficient number of cases to achieve satisfactory statistical power. A review of 26 publications containing information on 144 patients showed that patients with carcinoma confined to the EAC had similar survival times, regardless of the type of surgery performed (e.g., mastoidectomy, lateral TBR, and subtotal TBR) [17]. Patients with an extension of the disease into the middle ear had longer survival times if they received a subtotal TBR. On the other hand, the addition of radiation therapy to lateral TBR did not improve the survival rates [17]. Another multi-institutional review of 87 records that focused primarily on the

Table 3. Patterns of failure for patients with external auditory canal and middle ear cancer according to the RT type

\begin{tabular}{lccc} 
Recurrence site & $\begin{array}{c}\text { All patients } \\
(\mathbf{n}=\mathbf{3 0})\end{array}$ & $\begin{array}{c}\text { Postoperative RT } \\
(\mathbf{n}=\mathbf{2 0})\end{array}$ & $\begin{array}{c}\text { Definitive RT } \\
(\mathbf{n}=9)\end{array}$ \\
Any recurrence & 15 & 11 & 4 \\
Local failure & 4 & 3 & 1 \\
Regional failure & 1 & 0 & 1 \\
Distant & 10 & 8 & 2 \\
Lung & 7 & 6 & 1 \\
Spine & 2 & 1 & 1 \\
Bone & 1 & 1 & 0 \\
\hline
\end{tabular}

$\mathrm{RT}$, radiotherapy. 
roles of surgery and RT in these patients found that the 5-year OS and DFS rates for all patients were 55\% and 54\%, respectively [13]. Moreover, the $\mathrm{T}$ stage and treatment modality were significant prognostic factors. The 5-year DFS rates for the $\mathrm{T} 1, \mathrm{~T} 2$, and $\mathrm{T} 3$ patients were $83 \%, 45 \%$, and $0 \%$ in the RT group, and $75 \%, 75 \%$, and $46 \%$ in the surgery with $\mathrm{RT}$ group, respectively. They concluded that a radical RT is the treatment of choice for stage $\mathrm{T} 1$, and surgery combined with RT is recommended for $\mathrm{T} 2$ or $\mathrm{T} 3$ stage cancer. The role of chemotherapy, however, remains unclear [13].

Many authors have suggested that postoperative RT is effective for the control of residual tumors at incomplete resection margins $[3,4,18]$. The present study compared treatment outcomes between early stage and advanced stage tumors according to the treatment modality (e.g., postoperative and definitive RT). Although the results were not statistically significant, they suggested that the prognosis of patients with early stage tumors is more favorable than for patients with advanced stage tumors. Tumor extension is an important prognostic factor for patients with these cancers. These results also indicated that patients with early stage cancer achieve better outcomes with definitive RT rather than postoperative RT. The stage-related disease control rates for definitive and postoperative RT were $66.7 \%$ and $55.6 \%$, respectively. Therefore, this study suggests that patients with early stage cancer can undergo less invasive treatment. These results and conclusions are consistent with those of a previous report [19]. Some authors have suggested that small lesions without bony erosion can be treated with RT alone $[17,20]$. On the other hand, the disease control rates for advanced stage tumors treated with postoperative and definitive RT were 50\% and 37.5\%, respectively. Therefore, patients with advanced stage tumors show better outcomes when postoperative RT is used. Although the optimal radiation dose is unclear, the median doses for tumor control in this study were 59.4 Gy for postoperative RT and 68.4 Gy for definitive RT. A previous study indicated that total doses of 65 to 75 Gy are needed to control the disease [21].

One limitation of this study was that it was a retrospective, single institution study that included a 20-year time period. Heterogeneity among the patient characteristics (e.g., tumor histologic type) might have confounded the results. The most common histologic type of carcinoma of the EAC and middle ear is SCC. This type was also the most common type $(65.6 \%)$ in the present study population. ACC (28.1\%) was the second most common histologic type. ACC is a special entity among tumors of the EAC; it has a slow proliferation rate and a low tendency towards lymphatic spread. On the other hand, lesions recur frequently when they are not excised completely, which contributes to the poor prognosis of ACC patients [4]. Therefore, more aggressive surgery is recommended for patients with incompletely resected ACC and patients with late-stage diseases [19]. Paradoxically, this study revealed 5-year OS rates of $76.2 \%$ and $50 \%$ for patients with ACC and SCC, respectively. Secondly, although the MRI findings can be used to evaluate the parotid, soft tissue, infratemporal fossa, and temporal dura mater involvement [22], the patients in the present study were staged according to the clinical and CT-based radiologic findings so that the results would be comparable to those in other studies that used the Pittsburgh staging system. This staging system was applied to all histologic types of tumors. Thirdly, 14 patients $(46.7 \%)$ experienced treatment failure; the incidence of distant failure was particularly high. Further investigation will be needed to identify the role of chemotherapy for these patients. Physicians from several institutions have applied chemoradiation therapy (CRT) to improve the patients' prognosis. They found preoperative CRT to be effective $[3,22]$. The results of meta-analysis to assess the role of CRT for EAC SCC [15] suggested that preoperative CRT may improve the survival of surgically treated patients with EAC SCC. The meta-analysis results also suggested that definitive CRT may be an effective alternative to surgical resection.

Surgical approaches to treat advanced stage EAC and middle ear cancer often cause severe complications [17,23]. In addition, because the recurrence rates are high, a minimum of $66 \mathrm{~Gy}$ is recommended for patients with positive margins [4]. Newer treatment techniques, such as 3-dimensional conformal and intensity-modulated RT, can be used to deliver higher doses to the tumor and lower doses to the surrounding critical organs.

\section{Conclusion}

Patients with early stage EAC and middle ear carcinoma had better outcomes when definitive RT was used. Patients with advanced stage cancer experienced better outcomes with postoperative RT. The high rate of distant failure after RT, with or without surgery, reflected the lack of a consensus on the best therapeutic approach for treating carcinomas of the EAC and middle ear.

\section{Conflicts of Interest}

Conflict of interest relevant to this article was not reported. 


\section{References}

1. Koto M, Hasegawa A, Takagi R, Sasahara G, Ikawa H, Mizoe $\mathrm{JE}$, et al. Carbon ion radiotherapy for locally advanced squamous cell carcinoma of the external auditory canal and middle ear. Head Neck. 2016;38:512-6.

2. Kuhel WI, Hume CR, Selesnick SH. Cancer of the external auditory canal and temporal bone. Otolaryngol Clin North Am. 1996;29:827-52.

3. Nakagawa T, Kumamoto Y, Natori Y, Shiratsuchi H, Toh S, Kakazu Y, et al. Squamous cell carcinoma of the external auditory canal and middle ear: an operation combined with preoperative chemoradiotherapy and a free surgical margin. Otol Neurotol. 2006;27:242-8.

4. Pfreundner L, Schwager K, Willner J, Baier K, Bratengeier K, Brunner FX, et al. Carcinoma of the external auditory canal and middle ear. Int J Radiat Oncol Biol Phys. 1999;44:777-88.

5. Boamah H, Knight G, Taylor J, Palka K, Ballard B. Squamous cell carcinoma of the external auditory canal: a case report. Case Rep Otolaryngol. 2011;2011:615210.

6. Korzeniowski S, Pszon J. The results of radiotherapy of cancer of the middle ear. Int J Radiat Oncol Biol Phys. 1990;18:631-3.

7. Gabriele P, Magnano M, Albera R, Canale G, Redda MG, Krengli $\mathrm{M}$, et al. Carcinoma of the external auditory meatus and middle ear: results of the treatment of 28 cases. Tumori. 1994;80:40-3.

8. Waldemar E, Sorensen T, Bretlau P, Hansen HS. Cancer in the middle ear and the auditory canal. Ugeskr Laeger. 1995;157: 2139-42.

9. Spector JG. Management of temporal bone carcinomas: a therapeutic analysis of two groups of patients and long-term followup. Otolaryngol Head Neck Surg. 1991;104:58-66.

10. Chen WY, Kuo SH, Chen YH, Lu SH, Tsai CL, Cheng JC, et al. Postoperative intensity-modulated radiotherapy for squamous cell carcinoma of the external auditory canal and middle ear: treatment outcomes, marginal misses, and perspective on target delineation. Int J Radiat Oncol Biol Phys. 2012;82:148593.

11. Arriaga M, Curtin H, Takahashi H, Hirsch BE, Kamerer DB. Staging proposal for external auditory meatus carcinoma based on preoperative clinical examination and computed tomography findings. Ann Otol Rhinol Laryngol. 1990;99 (9 Pt 1):714-21.

12. Pemberton LS, Swindell R, Sykes AJ. Primary radical radio- therapy for squamous cell carcinoma of the middle ear and external auditory cana: an historical series. Clin Oncol (R Coll Radiol). 2006;18:390-4.

13. Ogawa K, Nakamura K, Hatano K, Uno T, Fuwa N, Itami J, et al. Treatment and prognosis of squamous cell carcinoma of the external auditory canal and middle ear: a multi-institutional retrospective review of 87 patients. Int J Radiat Oncol Biol Phys. 2007;68:1326-34.

14. Moody SA, Hirsch BE, Myers EN. Squamous cell carcinoma of the external auditory canal: an evaluation of a staging system. Am J Otol. 2000;21:582-8.

15. Takenaka Y, Cho H, Nakahara S, Yamamoto Y, Yasui T, Inohara $\mathrm{H}$. Chemoradiation therapy for squamous cell carcinoma of the external auditory canal: a meta-analysis. Head Neck. 2015;37:1073-80.

16. Prabhu R, Hinerman RW, Indelicato DJ, Morris CG, Werning JW, Vaysberg M, et al. Squamous cell carcinoma of the external auditory canal: long-term clinical outcomes using surgery and external-beam radiotherapy. Am J Clin Oncol. 2009;32: 401-4.

17. Prasad S, Janecka IP. Efficacy of surgical treatments for squamous cell carcinoma of the temporal bone: a literature review. Otolaryngol Head Neck Surg. 1994;110:270-80.

18. Kinney SE. Squamous cell carcinoma of the external auditory canal. Am J Otol. 1989;10:111-6.

19. Chang CH, Shu MT, Lee JC, Leu YS, Chen YC, Lee KS. Treatments and outcomes of malignant tumors of external auditory canal. Am J Otolaryngol. 2009;30:44-8.

20. Arthur K. Radiotherapy in carcinoma of the middle ear and auditory canal. J Laryngol Otol. 1976;90:753-62.

21. Hashi N, Shirato H, Omatsu T, Kagei K, Nishioka T, Hashimoto $S$, et al. The role of radiotherapy in treating squamous cell carcinoma of the external auditory canal, especially in early stages of disease. Radiother Oncol. 2000;56:221-5.

22. Gillespie MB, Francis HW, Chee N, Eisele DW. Squamous cell carcinoma of the temporal bone: a radiographic-pathologic correlation. Arch Otolaryngol Head Neck Surg. 2001;127: 803-7.

23. Leong SC, Youssef A, Lesser TH. Squamous cell carcinoma of the temporal bone: outcomes of radical surgery and postoperative radiotherapy. Laryngoscope. 2013;123:2442-8. 